\title{
Adhesion of Diazoquinone-Novolac Photoresist Films Implanted with Boron and Phosphorus Ions to Single-Crystal Silicon
}

- S. A. Vabishchevich,

- S. D. Brinkevich,

- D. I. Brinkevich \&

- V.S. Prosolovich

High Energy Chemistry volume 54, pages46-50 (2020)

\section{Abstract}

The effect of ion implantation on the specific energy of delamination of FP9120 diazoquinone-novolac photoresist films deposited on single-crystal silicon wafers has been studied. It has been found that during the implantation of boron and phosphorus ions, ester crosslinks between hydroxyl groups on the surface of an oxide layer of the silicon wafer and the carboxyl groups of 1-H-indene-3-carboxylic acid grafted to the polymer are formed at the photoresist-silicon interface, thereby leading to an increase in the specific energy $G$ of film delamination from the substrate. This effect is observed far beyond the ion range, being more pronounced in the case of implantation of phosphorus ions.

\section{REFERENCES}

1. 1

Kondyurin, A. and Bilek, M., Ion Beam Treatment of Polymers:

Application Aspects from Medicine to Space, Amsterdam: Elsevier, 2015, 2nd ed., p. 256.

\section{Google Scholar}

2. 2 
Odzhaev, V.B., Kozlov, I.P., Popok, V.N., and Sviridov, D.V., Ionnaya implantatsiya polimerov (Ion Implantation into Polymers), Minsk: Belgosuniversitet, 1998.

3. 3

Moreau, W.M., Semiconductor Lithography, New York: Plenum, 1988.

\section{Book Google Scholar}

4. 4

Brinkevich, D.I., Brinkevich, S.D., Vabishchevich, N.V., Odzhaev, V.B., and Prosolovich, V.S., Russ. Microelectron., 2014, vol. 43, no. 3, p. 194.

\section{CAS Article Google Scholar}

5. 5

Brinkevich, D.I., Kharchenko, A.A., Prosolovich, V.S., Odzhaev, V.B., Brinkevich, S.D., and Yankovskii, Yu.N., Russ. Microelectron, 2019, vol. 48, no. 3, p. 197.

\section{CAS Article Google Scholar}

6. 6

Brinkevich, D.I., Prosolovich, V.S., Yankovskii, Yu.N., Vabishchevich, S.A., Vabishchevich, N.V., and Gaishun, V.E, Prib.Metody Izmer., 2016, vol. 7 , no. 1, p. 77.

\section{Article Google Scholar}

7. 7

Rosenfeld, L.G., Ritter, J.R., Lardner, T.J., and Lin, M.R., J. Appl. Phys., 1990, vol. 67, no. 7, p. 3291.

\section{Article Google Scholar}

8. 8 
Malzbender, J., Toonder, J.M.J., Balkenende, A.R., and de With, G., Mater. Sci. Eng. R., 2002, vol. 36, p. 47.

\section{Article Google Scholar}

9. 9

Brinkevich, D.I., Vabishchevich, N.V., and Prosolovich, V.S., Inorg. Mater., 2012, vol. 48, no. 8, p. 768.

\section{CAS Article Google Scholar}

10.10

Vabishchevich, S.A., Vabishchevich, N.V., Brinkevich, D.I., Prosolovich, V.S., and Brinkevich, S.D., Vestn. Polotsk. Gos. Univ., Ser. C: Fundam. Nauki, 2016, no. 12, p. 51.

11. 11

Brinkevich, S.D., Reztsov, I.A., and Shadyro, O.I., High Energy Chem., 2014, vol. 48, no. 5, p. 303.

\section{CAS Article Google Scholar}

12.12

Brinkevich, S.D., Maliborskii, A.Ya., Kapusto, I.A., Sverdlov, R.L., Grigor'ev, Yu.V., Ivashkevich, O.A., and Shadyro, O.I., High Energy Chem., 2019, vol. 53, no. 2, p. 147.

\section{CAS Article Google Scholar}

13.13

Brinkevich, S.D., Kuzmuk, D.A., Sverdlov, R.L., and Shadyro, O.I., High Energy Chem., 2019, vol. 53, no. 6, p. 472.

\section{Article Google Scholar}


Neta, P. and Steenken, S., The Chemistry of Phenols, Rappoport, Z., Ed., Chichester, Wiley, 2003, part 1, p. 1097.

15.15

Eksperimental'nye metody khimii vysokikh energii (Experimental Techniques in High Energy Chemistry) Mel'nikov, M.Ya., Ed., Moscow: Izd. MGU, 2009, p. 169.

16.16

Harchenko, A.A., Brinkevich, D.I., Brinkevich, S.D., Lukashevich, M.G., and Odzhaev, V.B., J. Surf. Invest.: X-ray, Synchrotron Neutron Tech., 2015, vol. 9, no. 1, p. 87.

\section{CAS Article Google Scholar}

17.17

Brinkevich, D.I., Kharchenko, A.A., Brinkevich, S.D., Lukashevich, M.G., Odzhaev, V.B., Valeev, V.F., Nuzhdin, V.I., and Khaibullin, R.I., J. Surf. Invest.: X-ray, Synchrotron Neutron Tech., 2017, vol. 11, no. 4, p. 801.

\section{CAS Article Google Scholar}

18.18

Harchenko, A.A., Brinkevich, D.I., Brinkevich, S.D., Lukashevich, M.G., and Odzhaev, V.B., J. Surf. Invest.: X-ray, Synchrotron Neutron Tech., 2015, vol. 9, no. 2, p. 371.

\section{CAS Article Google Scholar}

Download references

\section{Author information}

Affiliations

1. Polotsk State University, 21440o, Novopolotsk, Belarus S. A. Vabishchevich

2. Belarussian State University, 220050, Minsk, Belarus

S. D. Brinkevich, D. I. Brinkevich \& V. S. Prosolovich 
Corresponding author

Correspondence to $\underline{\underline{S} . \mathrm{D} \text {. Brinkevich. }}$

\section{Additional information}

Translated by V. Makhlyarchuk

Rights and permissions

Reprints and Permissions

About this article

Cite this article

Vabishchevich, S.A., Brinkevich, S.D., Brinkevich, D.I. et al. Adhesion of Diazoquinone-Novolac Photoresist Films Implanted with Boron and Phosphorus Ions to Single-Crystal Silicon. High Energy Chem 54, 46-50 (2020). https://doi.org/10.1134/S0018143920010129

- Received24 July 2019

- Revised24 July 2019

- Accepted12 August 2019

- Published28 February 2020

- Issue DateJanuary 2020

- DOI https://doi.org/10.1134/S0018143920010129

Keywords:

- diazoquinone-novolac photoresist

- ion implantation

- adhesion

- silicon 